\title{
Development of A Passively Powered Knee Exoskeleton for Squat Lifting
}

\author{
R.K.P.S. Ranaweera ${ }^{1}$, R.A.R.C. Gopura ${ }^{1}$, T.S.S. Jayawardena ${ }^{2}$ \\ Bionics Laboratory - Department of Mechanical Engineering ${ }^{I}$, Department of Textile and Clothing Technology ${ }^{2}$, \\ University of Moratuwa, Katubedda, Moratuwa 10400, Sri Lanka
}

\author{
G.K.I. Mann $^{3}$ \\ Faculty of Engineering and Applied Science ${ }^{3}$, Memorial University of Newfoundland, \\ 230 Elizabeth Ave, St. John's, NL A1B 3X9, Canada \\ E-mail:pubudur@uom.lk,gopurar@uom.lk,jaya@uom.lk,gmann@mun.ca \\ www.mrt.ac.lk
}

\begin{abstract}
This paper proposes a knee exoskeleton with passive-powering mechanism to provide power assistance to the knee joint during squat lifting of objects from the ground. It is designed to capture and store $20 \%$ of the biomechanical energy dissipated at the biological knee joint during decent phase and return the harnessed energy in the ascent phase in a squatting cycle. The effectiveness of the proposed system was verified by evaluating performance of key muscles of knee joint using surface electromyography (sEMG) signals. Statistical data from experiments revealed a reduction of peak root-mean-square averages of sEMG signals of knee extensor muscles by $30-40 \%$ during squatting.
\end{abstract}

Keywords: knee exoskeleton, biomechanical energy harvesting, passive mechanisms, squatting.

\section{Introduction}

Exoskeleton systems or robots are mainly developed focusing on two different user groups, namely differently-abled people and able-bodied people. ${ }^{1}$ According to literature, limited number of full lowerextremity exoskeletons or joint-level exoskeletons have been developed to support task or work related activities by providing power-assistance. ${ }^{1}$ The application areas are mostly limited to either locomotion assistance, tool holding or lift assistance. ${ }^{1}$ Although functionality of autonomous externally-powered robotic exoskeletons are satisfactory, the wearer's performance is notably compromised. ${ }^{2}$ In particular, such devices struggle to reduce the human effort. The main cause is the inability of the system to match the complex kinematic and kinetic requirements demanded by human biological joints. Here, limitations of the complementing technologies such as gear drives, actuators, sensors, and power sources resulted in formulation of bulky and heavy electromechanical systems that are slow in operation. ${ }^{2}$

Alternatively, recent research studies indicate that passive-dynamic powering systems are effective at metabolic cost reduction and enhancing overall human performance. ${ }^{3}$ The body-powered exoskeletons are energetically autonomous and soft lightweight transmissions provide superior biomechanical compliance. ${ }^{3}$ However, performance and endurance augmentation through biomechanical energy harvesting still remain an open challenge. A wearable energy harnessing and releasing system that is capable of recycling the waste mechanical energy dissipated in the human body can help reduce metabolic energy consumption during activities of daily living. ${ }^{3}$ Complementing limb muscles energetically will augment the joint strength and moreover enhances endurance limits of muscle fatigue. As a result, the exoskeleton wearer's productivity will improve over a period of time. 


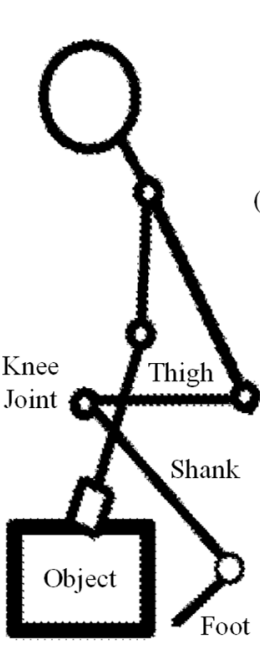

(A)

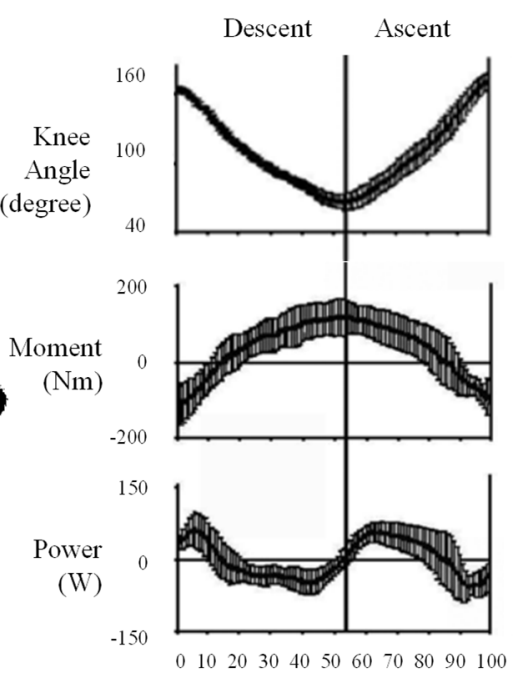

(B)

Percent Cycle
Fig. 1. Technique (A) and biomechanical data (B) of squatting (adapted from references 4 and 5).

In this context, a passively powered knee exoskeleton (PPKE) is developed to provide power assistance during squat lifting of objects from the ground (see Fig. 1(A)). The bio-inspired powering system is designed to recycle the waste biomechanical energy at the knee joint within the squatting cycle to help lift the wearer's own body mass. The knee exoskeleton is especially designed to pose no restrictions while walking and passively controlled locking mechanism only engages during a lifting task to capture and release biomechanical energy. The design considerations for the knee exoskeleton targeting squat lifting and walking are described in section 2. The design details of the exoskeleton and the working principle of passive powering mechanism is presented in section 3. The device was evaluated by measuring muscle performance of knee joint using surface muscle signals while performing semi-squats under different test conditions. The statistical data from experiments are analyzed in section 4.

\section{Design Considerations}

Load lifting is a typical industrial activity that is energy intensive. ${ }^{4}$ It involves moving an object from one position to another while traversing in vertical and/or horizontal directions. Considering the requirements for squatting as well as for walking, design considerations in relation to the knee joint were identified under functional and biomechanical points of view.

\subsection{Functional Considerations}

Among different lifting techniques the most commonly used approaches are squat lifting and stoop lifting. ${ }^{5}$ The knee exoskeleton is envisioned to assist the wearer's knee joint to lift moderately heavy loads. Hence squat or semisquat lifting is selected as the technique in focus for developing the proposed device, as it also promotes use of biomechanically more favorable approach. ${ }^{5}$

The device should essentially allow the wearer to ambulate freely in-between lifting tasks and pose no restriction for knee flexion during swing phase of human gait. The knee joint's limits of ranges of motion over the sagittal plane is measured to be $-10^{0}$ of hyper-extension and $140^{\circ}$ of flexion. ${ }^{4}$ According to gait data, ${ }^{6}$ maximum flexion of knee joint during swing phase is $60^{\circ}$.

\subsection{Biomechanical Considerations}

The muscle activity of thigh muscles are of interest when performing a squatting exercise. ${ }^{7}$ Notably, the quadriceps show greater muscle activity than the hamstrings during the complete ascent phase. ${ }^{4}$ The focus is to relieve muscle groups of the burden of having to concentrically and eccentrically work to control motion of the knee joint.

Biomechanical data during squatting (see Fig. 1(B)) were analyzed to identify the role of lower limb joints to generate the mechanical power for lifting. ${ }^{4}$ The notion is to recover the potential energy dissipated as a result of lowering the center of gravity of human body which henceforth referred to as passive energy. Here, the hip and knee joints dissipate energy through eccentric contraction of the main muscle groups on lower extremity during the decent phase ( 2 seconds period $){ }^{4}$ Similarly, during the ascending phase ( 2 seconds period), energy is generated while undergoing concentric contraction. ${ }^{4}$ In contrast, during initial stages $(0-15 \%$ cycle) of the decent phase, the knee flexor muscles act concentrically followed by extensor muscles acting eccentrically for the remainder of the descent (15-53\% cycle). ${ }^{4}$ The positive power is assumed to unlock the knee to initialize the descent. Throughout start of the ascending phase $(53-85 \%$ cycle) power is generated by knee extensor muscles by acting concentrically. ${ }^{4}$ However, towards end of the phase (85-100\%) knee flexor muscles started to dissipate energy gathered using knee flexors acting eccentrically. ${ }^{4}$ The negative power prevent undesirable level of hyperextension of knee joint. 
CAD Model of human subject $\left(50^{\text {th }}\right.$ percentile male)

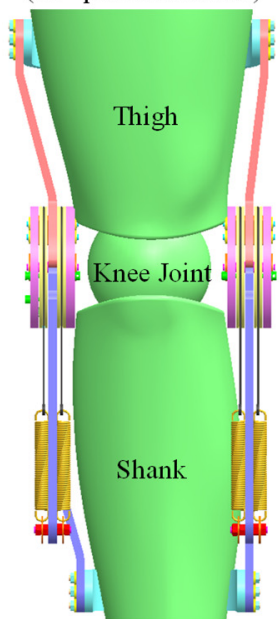

(A)

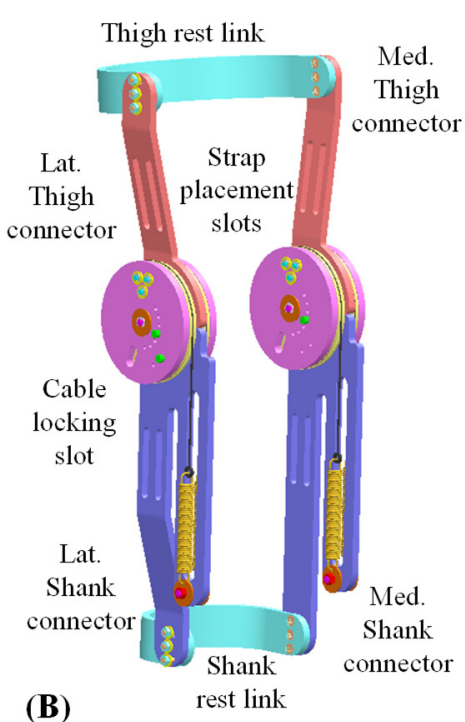

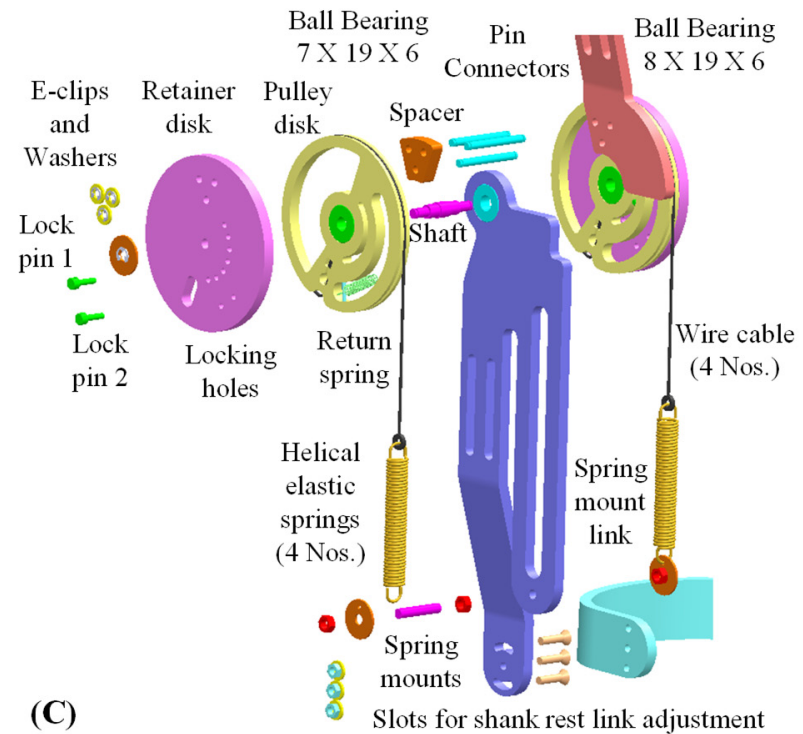

Fig. 2. CAD of PPKE on human model (A), passive-powering mechanism of PPKE (B), and exploded-view of PPKE (C).

\section{Design of Passively Powered Knee Exoskeleton}

Design objectives were to develop a simple, lightweight and affordable knee exoskeleton with good ergonomic conformance and offer power assistance for squat lifting while posing the minimal resistance for walking. Design specifications were derived for $50^{\text {th }}$ per. male population.

\subsection{Material Selection}

Acrylic glass, a thermoplastic polymer, is considered as the material of choice for developing the PPKE. Acrylic glass sheets can be easily cut in to various contours using computer numerically controlled laser cutting machines and formed in to complex shapes using thermoforming methods at an affordable cost. Acrylic is also considered to be non-toxic to the human skin and the environment friendly. Thus making it a suitable option that has not been previously explored in exoskeleton development. In order to counter the lower tensile strength of acrylic material (approx. $70 \mathrm{MPa}$ ), the design of the exoskeleton should be satisfactory. The flow of forces through the acrylic structure should be entirely compressive in nature. Bending or shear forces have been eliminated by effectively utilizing the symmetricity of the design.

\subsection{Structural System}

A computer-aided design model of the human was prepared using scanned data. The sizing and shaping of the structural system was then performed over the frontal and sagittal planes (see Fig. 2(A)). In order to avoid collision of thigh rest and shank rest at full squat position, shank connectors were designed with longer legs (see Fig. 2(B)). Thigh connectors were shorter in length to minimize interference at the medial side. The structure is held firmly on the limb segments using four Velcro straps placed through the linear slots on the connectors. Resting links can be easily replaced to accommodate varying limb cross-sections, if needed. The curved slots at the hinged end of the rest links provide the ability to adjust relative angular position of rests with the connectors. The slender acrylic structure assume the body shape over the frontal plane if contact forces are significantly high. The compliant structure hence reduce the possibility of internal joint injuries due to misalignments.

\subsection{Passive Powering Mechanism}

It is assumed that industrial workers are more likely to assume a semi-squat position during lifting tasks. Hence, the design has been planned for parallel-squat operation, where the knee bends approx. $70^{\circ}$ to $100^{\circ}$. In addition, the wearer need to have freedom to ambulate at normal walking speed ${ }^{6}(1.3 \mathrm{~m} / \mathrm{s})$. Consequently, the knee flexion should be left unconstrained up to $60^{\circ}$. The springs should engage to capture the negative energy when the knee flexes beyond this limit. Assuming the maximum knee angle happens up to $90^{\circ}$ in a regular squat lifting task, the effective angular displacement of $30^{\circ}$ can be utilized to energize a collection of linear elastic elements. 


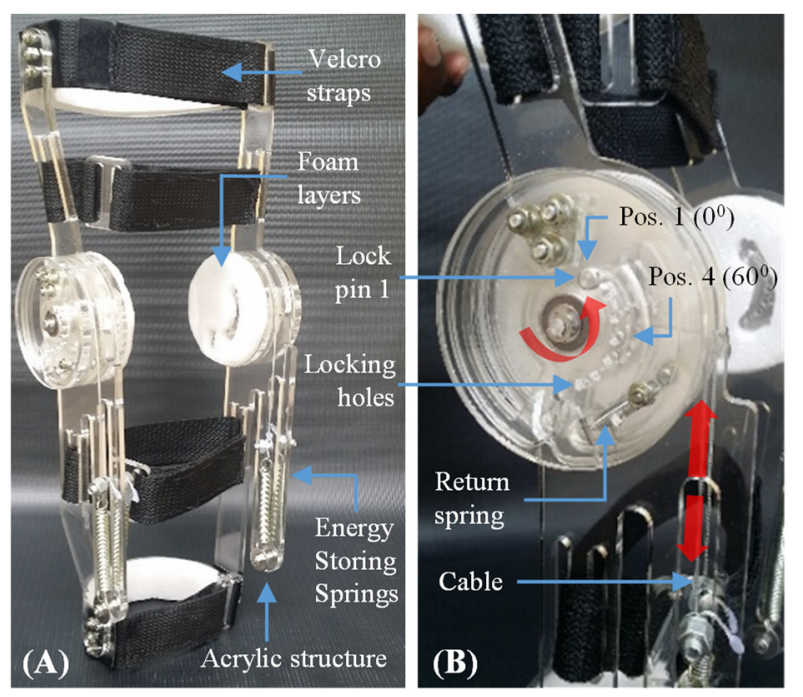

Fig. 3. Prototype of PPKE fabricated for the right limb (A) and close-up view of passive powering mechanism (B).

The proposed system ${ }^{8}$ to achieve this function is comprised of energy capturing/storing springs held parallel to shank, wire cables, pulley disks, smaller return springs and pins (see Fig. 2(C)). The fabricated prototype of PPKE also shows the key elements of the system (See Fig. 3(A)) with identical mechanisms bilaterally placed.

The working principal of the system ${ }^{8}$ is as follows. The pulley disks are fitted on bearings and are free to rotate about the axis of the shaft. However, by form the mechanical limits are in place to prevent rotation of the pulley in opposite or counter-clockwise direction. The clockwise rotation will be prevented by the smaller return springs that is held between the retainer disk and the pulley disk using set of pins. The lock pin 1 can be inserted in to a pulley disk locking hole that are spaced $20^{0}$ apart over a range of $0^{0}$ to $120^{\circ}$. If the lock pin is removed, the knee joint can be easily flexed without notable restriction from the exoskeleton. Here the stiffer springs act as a rigid body causing the flexible return springs to extend. As a result the pulley disk will rotate in a clockwise sense to allow the free length of the wire cable unchanged. When the knee traverses back to the rest position, the return spring will pull the pulley disk to its initial position. By placing the lock pin 1 in position number-one $\left(0^{0}\right)$ the pulley disk gets immediately locked with the retainer disk (see Fig. 3(B)). Consequently, when the knee gets flexed the stiffer springs starts to load gradually capturing energy of decent. If the lock pin is inserted in to position number-two $\left(20^{\circ}\right)$, the knee is free to flex up to the same limit and the pulley disk will come to a stop as the lock pin 1 traverses and reaches the mechanical limit over the curved slot. ${ }^{8}$ In order to allow unconstrained flexion during walking $(1.3 \mathrm{~m} / \mathrm{s})$, lock pin 1 should be inserted in position number-four $\left(60^{\circ}\right)$. As a result, during rest of the decent phase up to $90^{\circ}$, available passive energy is harnessed by the powering mechanism.

The spring selection is done to maximize the energy retention. However, considering the limited tensile strength of acrylic glass and the overall size of the device, the maximum force applied on a single spring is limited to $150 \mathrm{~N}$. The limits were determined by analyzing the stress distribution over the critically loaded acrylic components of the exoskeleton. In particular, the shank connectors (see Fig. 4(A)) and pulley disks (see Fig. 4(B)) were subjected to compression loadings and torsional loadings respectively. The finite element (FE) simulations provided an insight on the safety limits for the same. To accommodate manufacturing and material defects, the factor of safety (FOS) were maintained at 1.5 and above for all acrylic parts. Consequently, the structure and powering mechanisms are capable of withstanding heavy loads as a consequence of symmetrical positioning of passive elastic actuators.

The energetic response of the proposed passive energy recycling system can be mathematically modeled as follows. If a bilateral system with elastic helical springs having $5875 \mathrm{~N} / \mathrm{m}$ stiffness coefficient is used, the total strain energy stored in the system over a $30^{\circ}$ knee angle can be determined by (1),

$$
E=\frac{1}{2} k x^{2}
$$

Where, $k$ - stiffness coefficient $(\mathrm{N} / \mathrm{m}), x-$ spring extension (m) and $E$ - accumulated energy (J or Nm).
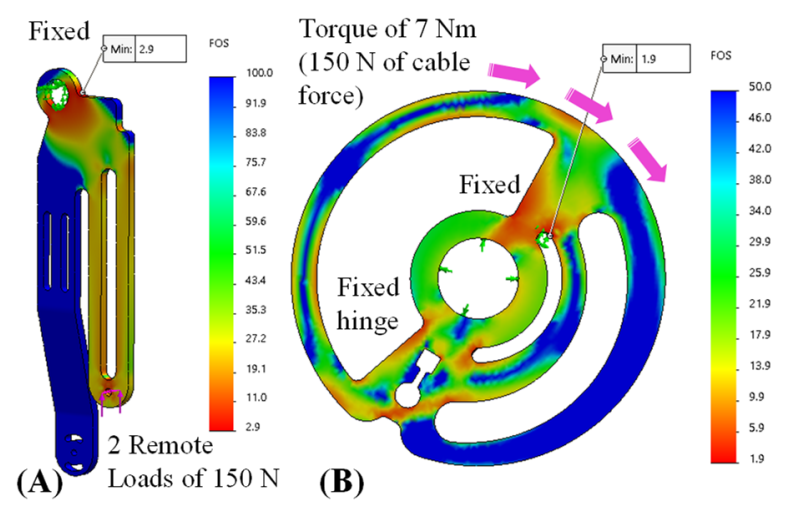

Fig. 4. Results of FE simulations: factory of safety (FOS) plot for lateral shank connector (A), and pulley disk (B). 


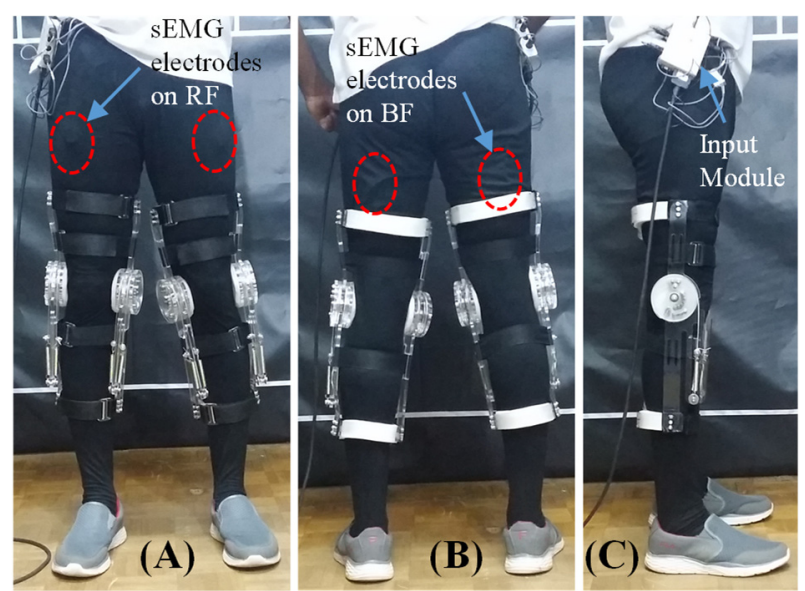

Fig. 5. Prototype of PPKE fitted on male subject with sEMG electrodes placed over muscle bellies of rectus femoris $(\mathrm{RF})$ and bicep femoris (BF) muscles: front (A), rear (B), and side (C).

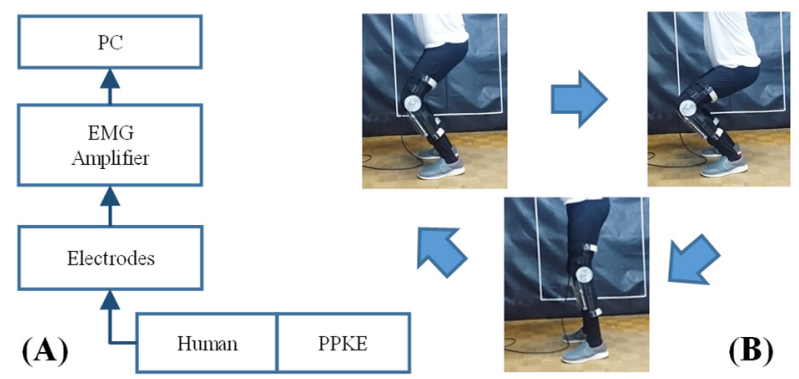

Fig. 6. Experimental set-up (A) and protocol (B) used for evaluation of PPKE performance.

The total energy dissipated and generated at the knee joint during decent and ascent phases from biomechanical studies were found to be $45 \mathrm{~J}$ and $50 \mathrm{~J}$ respectively. The selected springs thus have the capacity to collectively (eight springs on two PPKE units) capture and return approximately over $20 \%$ of total mechanical energy required considering loses due friction. Here, the maximum spring force is evaluated to be approx. $133 \mathrm{~N}$.

The overall mass of PPKE is approximately $900 \mathrm{~g}$ per unit. The acrylic sheets used for fabricating the prototype is $6 \mathrm{~mm}$ in thickness, the shafts and pins were made of aluminium alloys, and bearings, wire cables, springs and connectors were made of steel. In order to minimize the abrasion at the human-machine interface and weakening of contact during the entire range of motion a padded cushion made of polyethylene foam was affixed on the contact surfaces. Velcro strap located adjacent to the knee on the shank was mainly responsible for maintaining alignment of the axes of rotations of biological and exoskeleton knee joints.

\section{Evaluation of PPKE}

Experiments were performed to determine the possible benefits of using the passive energy recycling system to enhance strength and improve endurance limits of thigh muscles. According to the biomechanical studies the quadriceps muscle group, in particular the rectus femoris (RF) muscle, perform higher level of work to lift the body by undergoing concentric contraction during ascending phase. ${ }^{4}$ In addition, the RF is mainly responsible to lower the body gradually by undergoing eccentric contraction during descending phase. ${ }^{4}$ Consequently throughout the squatting cycle RF muscle activity is notably high., Since, the proposed powering mechanism include artificial muscles made of elastic springs that work in parallel to knee extensor muscles, the muscular workload is assumed to be shared between them. Thus, measurement of RF muscle activity based on surface electromyography (sEMG) signals will provide a realistic insight on the effectiveness of the proposed exoskeleton to relieve muscle effort. Similarly, bicep femoris (BF) muscle of hamstring muscle group is mainly responsible for unlocking the knee to initiate decent phase and to control the momentum of body during the ascend phase in a squatting cycle. ${ }^{4}$ Although the relative workload of $\mathrm{BF}$ muscle is comparatively lower than RF muscle, effect of the system on the knee flexor muscles can be appreciated by analyzing sEMG signals of BF muscle. Thus, sEMG signals of RF and BF muscles were used as two measures to objectively validate PPKE performance.

\subsection{Experimental Set-Up}

Wired sEMG electrodes connected to DELSYS Bagnoli EMG system were placed on muscle bellies of RF (see Fig. 5(A)) and BF (see Fig. 5(B)) muscles to measure electrical signals. The raw signals received by the Input Module (see Fig. 5(C)) were amplified using the Main EMG Amplifier and saved in the personal computer (See Fig. 6(A)). Here, sampling frequency was set at $2000 \mathrm{~Hz}$. The raw data is then post-processed using EMGworks software by DELSYS Inc. The root mean square (RMS) average of sEMG signals is calculated by (2),

$$
R M S \text { of } S E M G=\sqrt{\sum_{n=1}^{N} x_{n}^{2} / N}
$$

where, RMS, $x_{n}$ and $N$ are RMS of channel $\mathrm{n}(\mathrm{n}=1,2 .$.$) ,$ raw sEMG of channel $n$ and sample size, respectively. 
(A) Muscle activity of Rectus Femoris (RF) during Squat
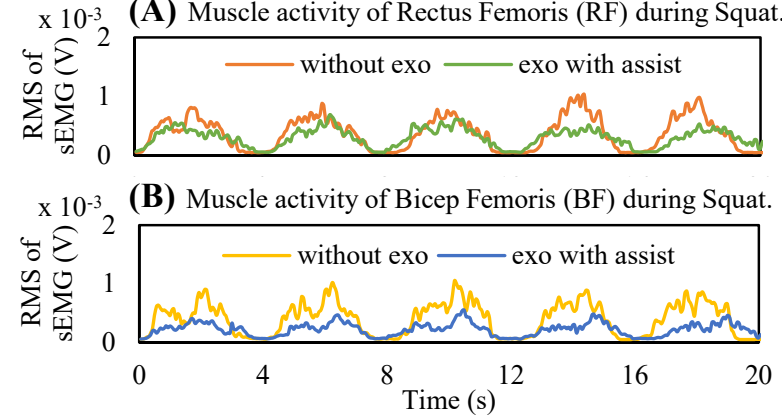

Fig 7. Muscle activity of rectus femoris (A) and bicep femoris (B) of right limb for a single subject during first 5 squat cycles.

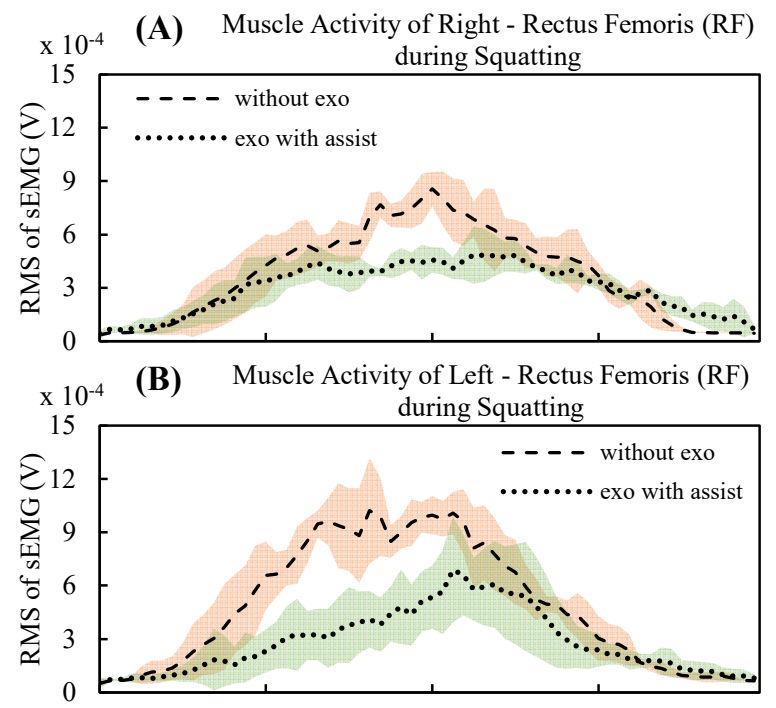

(C) Muscle Activity of Right - Rectus Femoris (RF)
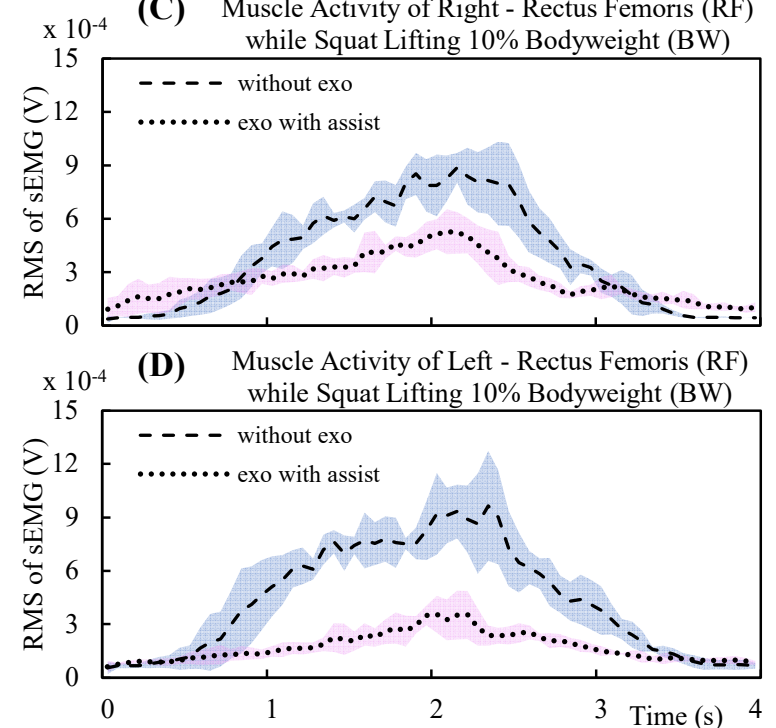

Fig. 8. Mean muscle activity of rectus femoris of right (A \& C) and left (B \& D) limbs for a single subject averaged over first 5 squat cycles. Band represents confidence interval of std. dev.

\subsection{Experimental Protocol}

The test protocols were developed to measure relative changes of muscle activity for both squatting as well as for squat lifting. List of tests conducted are as follows,

- $\quad$ squatting without exoskeleton

- $\quad$ squatting with power assistance of exoskeleton

- $\quad$ squat lifting $10 \%$ of bodyweight (BW) without exoskeleton

- $\quad$ squat lifting $10 \%$ of bodyweight (BW) with power assistance of exoskeleton

Each of the tests were conducted for a period of 100 seconds with an ascent-descent cycle of four seconds that ranged over $0^{0}$ to $100^{\circ}$ knee angle (See Fig. 6(B)). A metronome was followed to ensure consistent rate of squatting using visual feedback.

In order to determine sample size for the statistical analysis, a pilot study on squatting was performed with a single test subject. Here, RMS of sEMG signals over the first five squatting cycles ( 0 - 20 seconds) were analyzed for both RF (See Fig. 7(A)) and BF (See Fig. 7(B)) muscles. The means and standard deviations of the maximum amplitudes for with and without exoskeleton were determined and correlation coefficients between the paired data were calculated. According to standard practices, power-analysis for a paired-means test was performed using STATA, a statistical software package by StataCorp. Analysis outputted a sample size of 10 to meet a statically power of $80 \%$ and p-value of 0.05 . Subsequently, an experimental study was conducted with 10 able-bodied healthy male subjects to objectively validate the effectiveness of the PPKE performance foreseen in pilot study. The biometric data of the participants are as follows: age: $27 \pm 4$ years, weight: $60 \pm 9$ $\mathrm{kg}$, and height: $170 \pm 5.5 \mathrm{~cm}$.

\subsection{Results and Discussion}

The RMS of sEMG signals of each subject was averaged over five squatting cycles, namely 1-5, 6-10, $11-15,16-20$ and $21-25$ cycles. Since the amplitudes of RMS of sEMG signals tend to increase with increasing muscle fatigue, the five-cycle averaging approach provided broader view of muscle activity with statistical significance $(\mathrm{p}<0.05)$. In general, both $\mathrm{RF}$ and $\mathrm{BF}$ muscle activity were reduced when the power assistance of the exoskeleton is provided to the wearer. However, analysis of statistical data of RF muscles is of greater importance. 


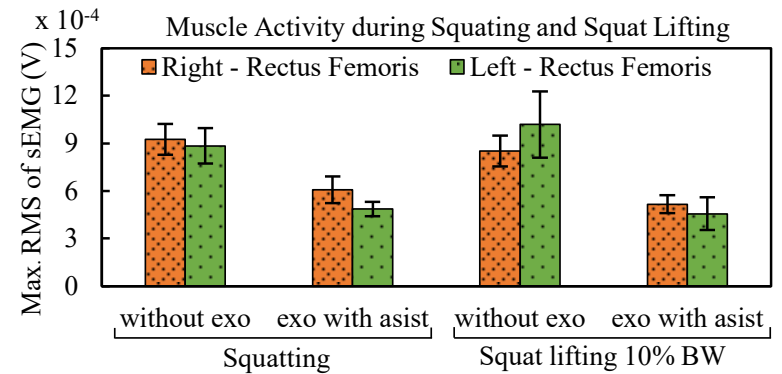

Fig. 9. Mean and standard error of mean of Max. RMS of sEMG of rectus femoris (RF) of right and left limbs over the first 5 squat cycles of 10 subjects.

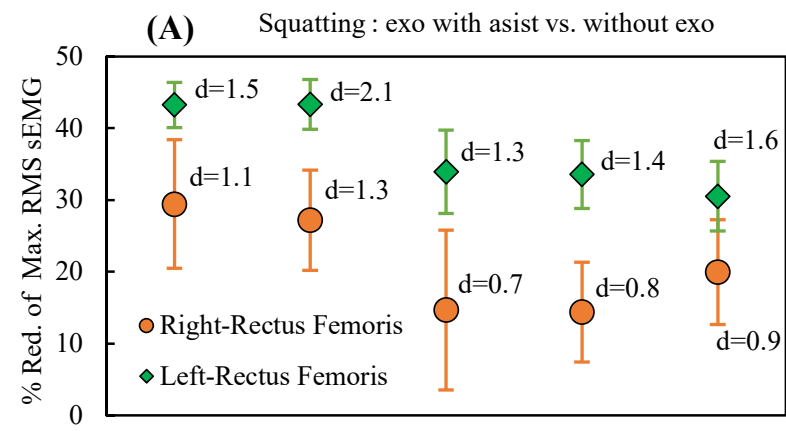

(B) Squat Lifting 10\% BW: Exo with asist vs. without exo

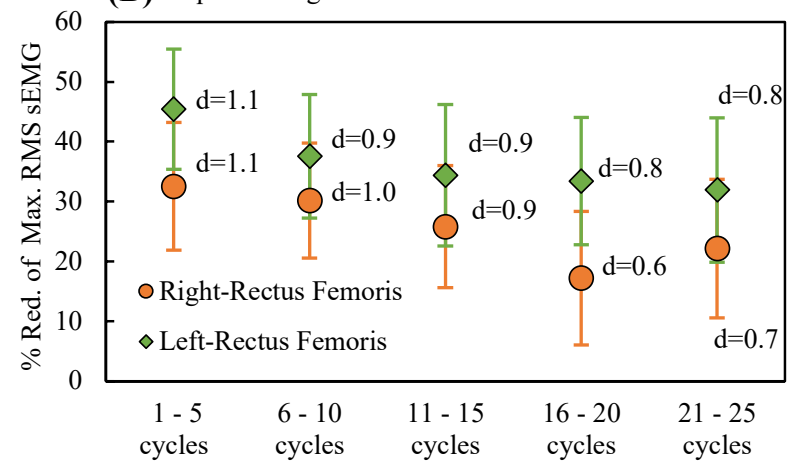

Fig. 10. Percent reduction of Max. RMS of sEMG of rectus femoris of right and left limbs over the first 5 squat cycles for 10 subjects during squatting (A) and squat lifting $10 \% \mathrm{BW}(\mathrm{B})$.

The mean muscle activity of RF of right and left limbs were computed for each subject for both squatting (See Fig. 8(A \& B)) and squat lifting (See Fig. 8(C \& D)). Here, a notable reduction of muscle activity is clearly visible when the power assistance of the exoskeleton is provided. The mean Max. RMS of sEMG signals of the 10 subjects provide a statistical confirmation on the positive effect of power assistance of the exoskeleton for squatting as well as for squat lifting (See Fig. 9). However, the standard error of mean (SEM) bars clearly indicate that the mean RF muscle activity between limbs is statistically insignificant (case of overlapping bars).
The percent reduction of Max. RMS of sEMG signals were determined for each of the averaged five-cycle periods for both squatting (See Fig. 10(A)) and squat lifting $10 \%$ of BW (See Fig. 10(B)). All percent reductions were evaluated to be statistically significant $(p<0.05)$. According to 'Cohen's d' the effect size in general was also evaluated to be 'large' $(\mathrm{d}>0.8)$. Hence, PPKE has effectively reduced peak muscle activity $30-$ $40 \%$ during the first five squatting cycles. Here, limb preference for load bearing (right) was identified to be the governing reason for asymmetry of the results.

\section{Conclusion}

PPKE is energetically autonomous, adaptable and nimble thus making it suitable for the industrial use. The prototype served as a proof of concept and effectively reduced muscle activity of rectus femoris muscles. Statistically, the effect size was determined as 'large'. As future work, clinical testing with metabolic cost estimation is considered to further improve the design.

\section{Acknowledgements}

Authors acknowledge Senate Research Council of University of Moratuwa (Grant No. SRC/CAP/2014/08).

\section{References}

1. B. Chen et al., "Recent developments and challenges of lower extremity exoskeletons," J. Orthop. Transl., vol. 5, pp. 26-37, 2016.

2. A. J. Young and D. P. Ferris, "State of the art and future directions for lower limb robotic exoskeletons," Trans. Neur. Syst. Rehab. Eng., vol. 25, no. 2, pp. 171-182, 2017.

3. S. H. Collins et al., "Reducing the energy cost of human walking using an unpowered exoskeleton," Nature, vol. 522, no. 7555, pp. 212-215, 2015.

4. D. G. E. Robertson et al., "Lower extremity muscle functions during full squats," J. Appl. Biomech., vol. 24, no. 4, pp. 333-339, 2008.

5. S. Hwang et al., "Lower extremity joint kinetics and lumbar curvature during squat and stoop lifting," $B M C$ Musculoskelet. Disord., vol. 10, no. 1, p. 15, 2009.

6. B. R. Umberger and P. E. Martin, "Mechanical power and efficiency of level walking with different stride rates," $J$. Exp. Biol., vol. 210, no. 18, pp. 3255-3265, 2007.

7. J. P. Pollard et al., "Forces and moments on the knee during kneeling and squatting," J. Appl. Biomech., vol. 27, no. 3, pp. 233-241, 2011.

8. Pubudu Ranaweera, Passively Powered Knee Exoskeleton-ICAROB 2018. 2018 [Online]. Available: https://www.youtube.com/watch? $=$ =ZZC1cgQo8H4.

[Accessed: 24- Apr- 2018] 